\title{
Fracturas Femorais Atípicas e o Tratamento com Bifosfonatos: É um Factor de Risco?
}

\author{
Atypical Femoral Fractures and Bisphosphonates Treatment: Is it a Risk \\ Factor?
}

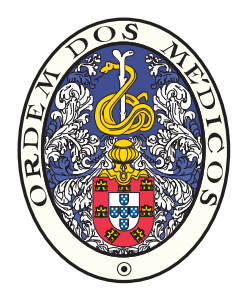

\author{
Nuno GEADA ${ }^{1}$, Inês MAFRA ${ }^{1}$, Rogério BARROSO ${ }^{1}$, José FRANCO ${ }^{1}$ \\ Acta Med Port 2014 Nov-Dec;27(6):704-709
}

RESUMO

Introdução: Os bifosfonatos têm eficácia comprovada na prevenção das fracturas osteoporóticas. Contudo, têm sido descritas fracturas femorais atípicas associadas à toma prolongada de bifosfonatos com um padrão radiológico bem definido. Os objectivos no nosso estudo foram caracterizar os doentes de idade $\geq 65$ anos com fracturas femorais (subtrocantéricas/diafisárias) consideradas típicas e atípicas e avaliar a relação entre a ocorrência das fracturas atípicas e o uso de bifosfonatos.

Material e Métodos: Realizámos um estudo caso-controlo com os doentes admitidos no nosso Hospital por fractura subtrocantérica ou diafisária do fémur, num período de cinco anos e meio. Após aplicação dos critérios de exclusão, as 92 fracturas (91 doentes) foram classificadas como atípicas ou típicas. A determinação do tratamento prévio com bifosfonatos foi obtida através do historial clínico dos doentes.

Resultados: Foram encontradas 11 fracturas atípicas (10 doentes) e 81 fracturas típicas (81 doentes). A idade mediana entre os dois grupos foi estatisticamente diferente (72 anos - atípicas vs 80 anos - típicas, $p<0,01)$. A razão do uso de bifosfonatos foi de 0,60 nas fracturas atípicas e de 0,01 nas típicas, traduzindo um odds ratio de 101,1 $(p<0,01)$.

Discussão: Na generalidade, os nossos resultados são apoiados e estão de acordo com os estudos publicados referentes à ocorrência de fracturas femorais atípicas associadas à toma de bifosfonatos.

Conclusão: Apesar do pequeno número de casos foi possível demonstrar a relação, estatisticamente, significativa entre as fracturas femorais atípicas e a toma de bifosfonatos. De notar que estas fracturas atípicas ocorreram em doentes, significativamente, mais jovens do que os doentes com fracturas típicas.

Palavras-chave: Difosfonatos; Fracturas do Fémur; Factores de Risco.

\section{ABSTRACT}

Introduction: Bisphosphonates are effective in preventing osteoporotic fracture, however atypical femoral fractures with a well-defined radiological pattern have been described in association with prolonged treatment with bisphosphonates. Our objectives in this study were to characterize patients $\geq 65$ years old with femoral fractures (subtrochanteric/diaphyseal) considered typical and atypical and the relationship between the occurrence of atypical fractures and bisphosphonate use.

Material and Methods: We conducted a case-control study with patients admitted in our hospital with subtrochanteric or diaphyseal femur fracture in a period of five and a half years. After applying the exclusion criteria, the 92 fractures (91 patients) were classified as typical or atypical. The determination of prior bisphosphonate treatment was obtained through consultation of the individual medical history.

Results: We found 11 atypical fractures (10 patients) and 81 typical fractures (81 patients). The median age of both groups was statistically different (72 years - atypical vs. 80 years - typical, $p<0.01)$. The reason for the use of bisphosphonates was 0.60 in atypical fractures and 0.01 in typical, and an odds ratio of 101.1 was obtained $(p<0.01)$.

Discussion: Our results are supported and are in agreement with published studies relating to the occurrence of atypical femoral fractures associated with treatment with bisphosphonates.

Conclusion: Despite the small number of cases it was possible to demonstrate a statistically significant relation between atypical femoral fractures and treatment with bisphosphonates. One should note that these atypical fractures occurred in patients significantly younger than patients with typical fractures.

Keywords: Diphosphonates; Femoral Fractures; Risk Factors.

\section{INTRODUÇÃO}

Os bifosfonatos têm eficácia comprovada no tratamento da osteoporose e prevenção das fracturas osteoporóticas típicas. ${ }^{1-3}$ Contudo, têm vindo a ser descritos casos de fracturas femorais atípicas (com localização subtrocantérica ou diafisária) e publicados estudos que as associam à toma prolongada de bifosfonatos, ${ }^{4-6}$ nomeadamente acima dos cinco anos, ${ }^{7}$ por eventual insuficiência de capacidade regenerativa do osso. ${ }^{8,9}$ Existem mesmo estudos que relacionam as fracturas femorais atípicas a tomas menos prolongadas ( $<2$ anos). ${ }^{5} \mathrm{~A}$ insuficiência da capacidade regenerativa do osso parece, também, afectar a consolidação da fractura levando em alguns casos à necessidade de tratamentos subsequentes. ${ }^{9}$

Estas fracturas femorais atípicas resultam de um trauma minor e têm um padrão radiológico bem definido: traço de fractura com orientação transversal, espessamento focal da cortical externa, espícula interna e mínima cominução. ${ }^{8,10}$

Este é um tema controverso uma vez que também existem estudos que demonstraram não haver associação entre a toma de bifosfonatos e as fracturas femorais atípicas. ${ }^{11}$

1. Serviço de Ortopedia. Centro Hospitalar Barreiro-Montijo. Barreiro. Portugal.

Recebido: 29 de Dezembro de 2013 - Aceite: 19 de Março de 2014 | Copyright $\odot$ Ordem dos Médicos 2014 
À data de escrita deste trabalho foram apenas encontrados trabalhos portugueses com descrição de casos clínicos individuais, o que não permite estudar a dimensão do problema na nossa realidade.

Os objectivos deste trabalho são definir as características dos doentes de idade igual ou superior a 65 anos, com fracturas femorais (subtrocantéricas e diafisárias) consideradas atípicas e típicas e avaliar a relação entre a ocorrência das fracturas atípicas e o uso de bifosfonatos.

\section{MATERIAL E MÉTODOS}

\section{Desenho de estudo e população}

Realizámos um estudo caso-controlo com todos os doentes com idade igual ou superior a 65 anos, admitidos no nosso Hospital com fractura subtrocantérica ou diafisária do fémur, entre 1 de Janeiro de 2008 e 30 de Junho de 2013 (dados recolhidos entre 17 de Julho de 2013 e 2 de Setembro de 2013).

Foram excluídos doentes com fracturas resultantes de alta energia (acidente de viação ou queda superior à própria altura), fracturas iterativas (peri-implante) ou doentes com antecedentes neoplásicos conhecidos.

\section{Fonte dos dados}

Os eventos fractura subtrocantérica ou diafisária do fémur foram obtidos através da pesquisa no Gabinete de Codificação Clínica do nosso Hospital, respectivamente, pelos códigos 82022 e 82101 da Classificação Internacional de Doenças - $9^{a}$ Revisão. Posteriormente, os eventos e o tipo de fractura foram confirmados através da consulta do processo clínico e observação do radiograma digital à data da fractura. A consulta do processo clínico foi efectuada no Arquivo Clínico do nosso Hospital e através do programa informático Sistema de Apoio ao Médico (SAM); a observação dos radiogramas digitais foi realizada no programa informático CARESTREAM Vue PACS, que serve de arquivo imagiológico no nosso Hospital desde 2008, razão pela qual o estudo tem a sua data de início nesse ano.

\section{Definição de casos e controlos}

Noventa e um doentes cumpriram os critérios de inclusão, tendo sido as fracturas subtrocantéricas $(n=80)$ ou fracturas diafisárias do fémur $(n=12)$ classificadas como sendo fracturas atípicas (casos) ou típicas (controlos) de acordo com a observação dos radiogramas digitais realizada pelos autores, sem conhecimento prévio dos antecedentes pessoais ou medicação habitual de cada doente. Em caso de diferença na avaliação dos radiogramas, a conclusão obtida foi através de consenso entre os autores.

A classificação das fracturas como atípicas (casos) foi realizada com base nos critérios revistos de definição das fracturas femorais atípicas publicados pela American Society for Bone and Mineral Research ${ }^{8}$ : trauma mínimo ou ausência de trauma, fractura predominantemente transversal, espessamento focal da cortical externa, espícula interna e mínima cominução. Foram classificadas como típicas (controlos) as fracturas que não se incluíam nos critérios atrás definidos.

\section{Avaliação de exposição}

A determinação de tratamento prévio com bifosfonato à data da fractura foi obtida através de entrevista directa ao doente, consulta do processo clínico, histórico de prescrição local no SAM e/ou histórico de prescrição nacional mediante consulta da Plataforma de Dados da Saúde. Nos doentes sob tratamento com bifosfonatos foram obtidos os dados relativos ao fármaco específico, a data de início de tratamento e sua manutenção.

\section{Análise estatística}

Realizámos uma análise descritiva dos dois grupos (idade, sexo, tipo de fractura e tratamento com bifosfonatos) utilizando a base de dados registada no programa Microsoft Excel ${ }^{\circledR}$.

Comparámos a idade mediana de ambos os grupos usando o teste de Wilcoxon.

Para avaliar a associação entre a ocorrência das fracturas atípicas e o uso dos bifosfonatos calculámos o odds ratio através do teste exacto de Fisher, de acordo com as características da nossa população em estudo. ${ }^{12}$

As diferenças entre grupos foram consideradas estatisticamente significativas quando obtidos valores de $p$ inferior a 0,05 .

Os cálculos dos testes de Wilcoxon e odds ratio foram realizados utilizando o programa $\mathrm{R}$, versão 3.0 .2 (http:// www.R-project.org/). ${ }^{13}$

\section{RESULTADOS}

Dos 112 doentes com idade igual ou superior a 65 anos, internados entre 1 de Janeiro de 2008 e 30 de Junho de 2013 com o diagnóstico de fractura subtrocantérica ou diafisária do fémur, 91 doentes cumpriram os critérios de inclusão, o que corresponde a 92 fracturas (Fig. 1). Destas foram encontradas 11 fracturas atípicas, em 10 doentes (um caso de fractura bilateral simultânea) e 81 fracturas típicas, em 81 doentes. Todas as fracturas foram tratadas com cavilha endomedular.

O grupo das fracturas atípicas apresentou três fracturas diafisárias e oito fracturas subtrocantéricas. Neste grupo, a mediana de idades foi de 72 anos (65-80), sendo composto apenas por doentes do sexo feminino (Tabela 1). O grupo das fracturas típicas apresentou nove fracturas diafisárias e 72 fracturas subtrocantéricas. Neste grupo a mediana de idades foi de 80 anos (65-99), sendo composto, maioritariamente, por doentes do sexo feminino $(83,9 \%)$. A idade mediana entre os dois grupos foi estatisticamente diferente (72 vs 80 anos, $p<0,01$ ).

O uso de bifosfonatos foi registado em seis doentes com fracturas atípicas, com uma mediana de duração de tratamento de cinco anos. Nos restantes quatro doentes com fracturas atípicas não havia registo da toma de bifosfonatos. No caso das fracturas típicas o uso de bifosfonatos foi registado em apenas um doente. (Tabela 2). 


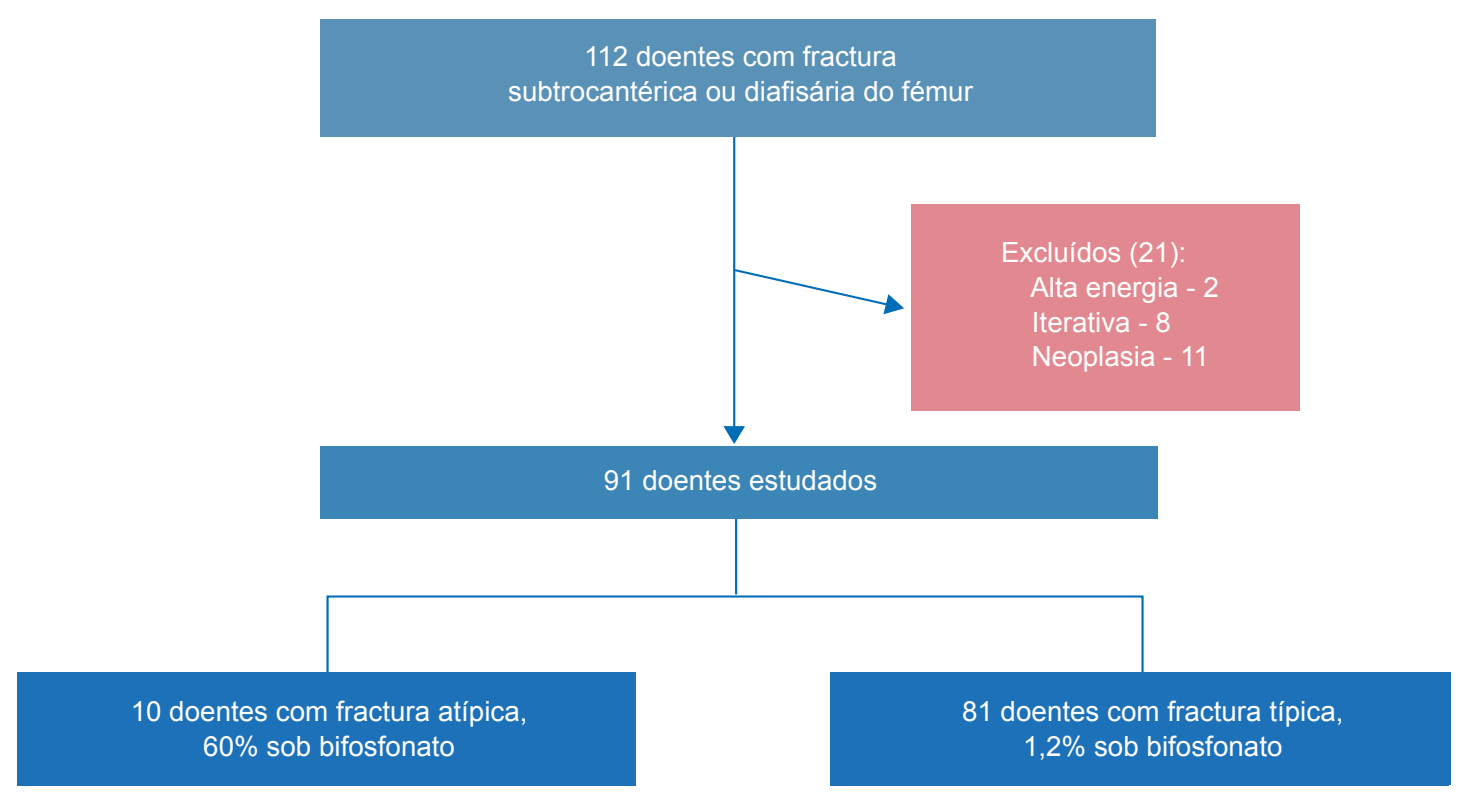

Figura 1 - Explicação da escolha dos doentes estudados com diagnóstico de fractura subtrocantérica ou diafisária do fémur

Tabela 1 - Descrição dos doentes por fractura femoral típica e atípica

\begin{tabular}{|c|c|c|}
\hline \multirow[b]{2}{*}{ Variável } & \multicolumn{2}{|c|}{ Doentes com fractura, $n(\%)$} \\
\hline & $\begin{array}{l}\text { Atípica } \\
(n=10)\end{array}$ & $\begin{array}{c}\text { Típica } \\
(n=81)\end{array}$ \\
\hline \multicolumn{3}{|l|}{ Lado } \\
\hline Direito & $2(20,0)$ & $40(49,4)$ \\
\hline Esquerdo & $7(70,0)$ & $41(50,6)$ \\
\hline Bilateral & $1(10,0)$ & $0(0,0)$ \\
\hline Uso de bifosfonatos & $6(60,0)$ & $1(1,2)$ \\
\hline Alendronato & $6(100,0)$ & $1(100,0)$ \\
\hline \multicolumn{3}{|c|}{ Duração do tratamento com bifosfonatos (anos) } \\
\hline Sem uso & $4(40,0)$ & $0(0,0)$ \\
\hline$<5$ & $2(20,0)$ & $1(1,2)$ \\
\hline $5-10$ & $3(30,0)$ & $0(0,0)$ \\
\hline$>10$ & $1(10,0)$ & $0(0,0)$ \\
\hline Sexo feminino & $10(100,0)$ & $68(83,9)$ \\
\hline \multicolumn{3}{|l|}{ Idade (anos) } \\
\hline $65-69$ & $3(30,0)$ & $7(8,6)$ \\
\hline $70-74$ & $5(50,0)$ & $16(19,8)$ \\
\hline $75-79$ & $1(10,0)$ & $14(17,3)$ \\
\hline $80-84$ & $1(10,0)$ & $20(24,7)$ \\
\hline $85-89$ & $0(0,0)$ & $19(23,5)$ \\
\hline $90-95$ & $0(0,0)$ & $1(1,2)$ \\
\hline $95-99$ & $0(0,0)$ & $4(4,9)$ \\
\hline
\end{tabular}

Em todos os casos o alendronato era o bifosfonato prescrito. A razão do uso de bifosfonatos no caso fracturas atípicas foi de 0,60 e do uso de bifosfonatos no caso de fracturas típicas foi de 0,01 . Obtivemos um odds ratio de 101,1
(Intervalo de Confiança de 95\%; 9,5 a 5326,2; $p<0,01$ ), resultado que é estatisticamente significativo.

Numa segunda análise com ajustamento para os doentes com divisão entre toma de bifosfonatos $<5$ anos ou 
Tabela 2 - Descrição dos doentes com fractura femoral típica e atípica, segundo a toma ou não de bifosfonatos

\begin{tabular}{|c|c|c|c|c|}
\hline & \multicolumn{2}{|c|}{$\begin{array}{l}\text { Doentes com fractura atípica } \\
\qquad(n=10)\end{array}$} & \multicolumn{2}{|c|}{$\begin{array}{l}\text { Doentes com fractura típica } \\
\qquad(n=81)\end{array}$} \\
\hline & $\begin{array}{l}\text { Uso de bifosfonatos, } \\
\qquad \begin{array}{c}n(\%) \\
(n=6)\end{array}\end{array}$ & $\begin{array}{l}\text { Sem uso de bifosfonatos, } \\
\qquad \begin{array}{c}n(\%) \\
(n=4)\end{array}\end{array}$ & $\begin{array}{l}\text { Uso de bifosfonatos, } \\
\qquad \begin{array}{c}n(\%) \\
(n=1)\end{array}\end{array}$ & $\begin{array}{l}\text { Sem uso de bifosfonatos, } \\
\qquad \begin{array}{c}\boldsymbol{n}(\%) \\
(n=80)\end{array}\end{array}$ \\
\hline Sexo feminino & $6(100,0)$ & $4(100,0)$ & $1(100,0)$ & $67(83,8)$ \\
\hline \multicolumn{5}{|l|}{ Idade } \\
\hline $65-69$ & $3(50,0)$ & $0(0,0)$ & $0(0,0)$ & $7(8,8)$ \\
\hline $70-74$ & $3(50,0)$ & $2(50,0)$ & $0(0,0)$ & $16(20,0)$ \\
\hline $75-79$ & $0(0,0)$ & $1(25,0)$ & $0(0,0)$ & $14(17,5)$ \\
\hline $80-84$ & $0(0,0)$ & $1(25,0)$ & $1(100,0)$ & $19(23,3)$ \\
\hline $85-89$ & $0(0,0)$ & $0(0,0)$ & $0(0,0)$ & $19(23,3)$ \\
\hline $90-95$ & $0(0,0)$ & $0(0,0)$ & $0(0,0)$ & $1(1,3)$ \\
\hline $95-99$ & $0(0,0)$ & $0(0,0)$ & $0(0,0)$ & $4(5,0)$ \\
\hline Uso de corticóides & $0(0,0)$ & $2(50,0)$ & $0(0,0)$ & $1(1,3)$ \\
\hline
\end{tabular}

Tabela 3 - Descrição dos doentes com fractura femoral típica e atípica, segundo a toma de bifosfonatos $<5$ ou $\geq 5$ anos

\begin{tabular}{|c|c|c|c|c|}
\hline & \multicolumn{2}{|c|}{$\begin{array}{l}\text { Doentes com fractura atípica } \\
\qquad(n=10)\end{array}$} & \multicolumn{2}{|c|}{$\begin{array}{l}\text { Doentes com fractura típica } \\
\qquad(n=81)\end{array}$} \\
\hline & $\begin{array}{l}\text { Uso de bifosfonato } \\
\geq 5 \text { anos, } \\
n(\%) \\
(n=4)\end{array}$ & $\begin{array}{c}\text { Sem uso/Uso de } \\
\text { bifosfonato }<5 \text { anos, } \\
n(\%) \\
(n=6)\end{array}$ & $\begin{array}{c}\text { Uso de bifosfonato } \\
\geq 5 \text { anos, } \\
n(\%) \\
(n=0)\end{array}$ & $\begin{array}{c}\text { Sem uso/Uso de } \\
\text { bifosfonato }<5 \text { anos, } \\
n(\%) \\
(n=81)\end{array}$ \\
\hline Sexo feminino & $4(100,0)$ & $6(100,0)$ & $0(100,0)$ & $68(83,9)$ \\
\hline \multicolumn{5}{|l|}{ Idade } \\
\hline $65-69$ & $3(75,0)$ & $0(0,0)$ & - & $7(8,6)$ \\
\hline $70-74$ & $1(25,0)$ & $4(66,7)$ & - & $16(19,8)$ \\
\hline $75-79$ & $0(0,0)$ & $1(16,7)$ & - & $14(17,3)$ \\
\hline $80-84$ & $0(0,0)$ & $1(16,7)$ & - & $20(24,7)$ \\
\hline $85-89$ & $0(0,0)$ & $0(0,0)$ & - & $19(23,5)$ \\
\hline $90-95$ & $0(0,0)$ & $0(0,0)$ & - & $1(1,2)$ \\
\hline $95-99$ & $0(0,0)$ & $0(0,0)$ & - & $4(4,9)$ \\
\hline
\end{tabular}

$\geq 5$ anos (Tabela 3 ), obtivemos odds ratio igual a infinito (Intervalo de Confiança de 95\%; 6,76 a infinito; $p<0,01$ ), resultado que é estatisticamente significativo.

A presença de sintomas prodrómicos estava apenas descrito numa doente de 65 anos, sob tratamento prolongado com bifosfonatos (> 10 anos) e com fractura atípica bilateral simultânea. (Fig. 2).

\section{DISCUSSÃO}

Em termos gerais, os resultados do nosso estudo estão de acordo com os estudos publicados referentes à ocorrência de fracturas femorais atípicas.

As fracturas atípicas representaram no nosso estudo $11 \%$ das fracturas subtrocantéricas e diafisárias do fémur, no período estudado. Este valor é semelhante ao encontrado por Thompson et al, ${ }^{4}$ Meier et $\mathrm{al}^{5}$ e Park-Wyllie et al, ${ }^{7}$ respectivamente com valores de $7 \%, 8 \%$ e $11 \%$. 

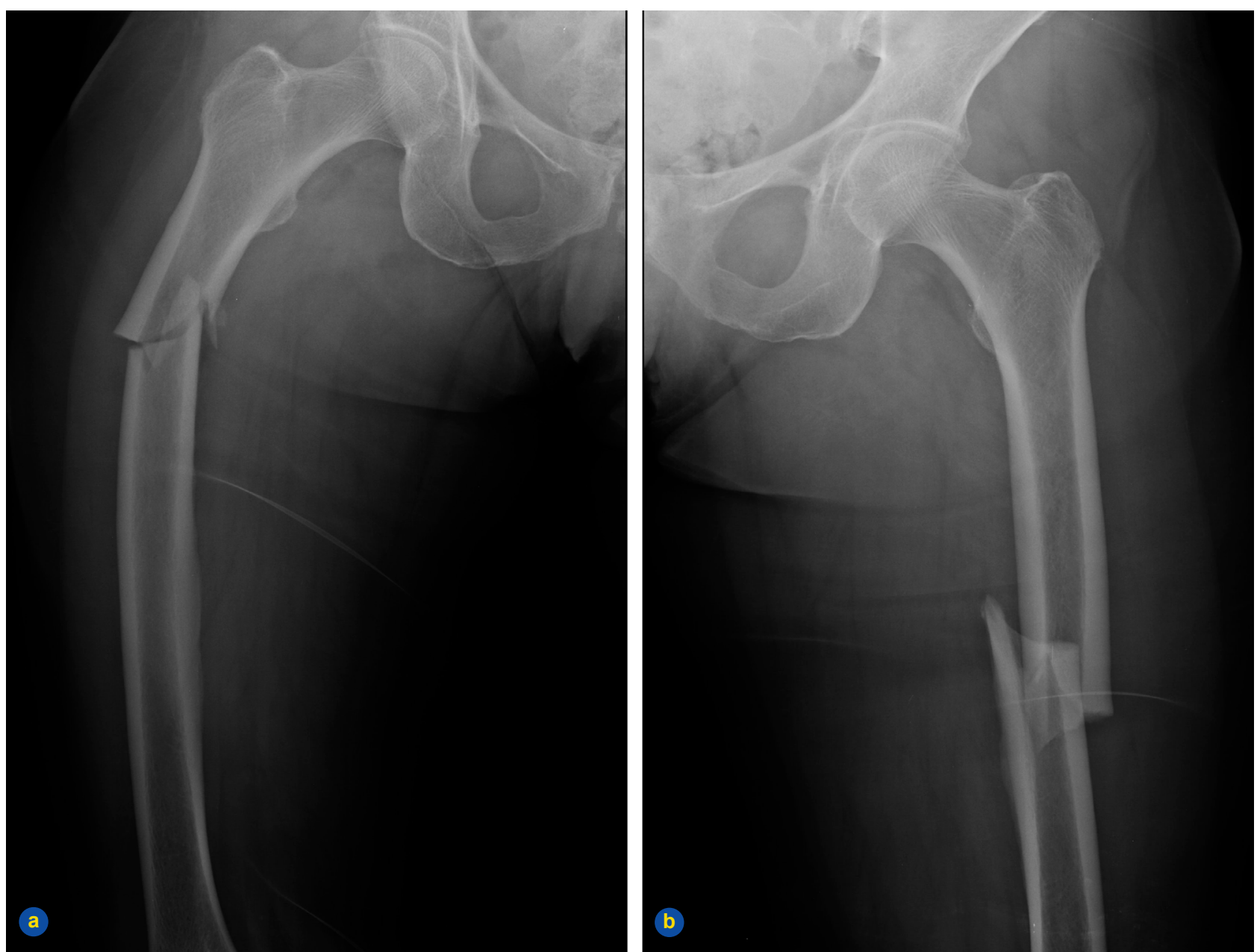

Figura 2 - Radiogramas da coxa direita (A) e esquerda (B) em doente com fractura bilateral simultânea e sob tratamento com bifosfonatos. De notar a presença de características atípicas em ambas as fracturas.

A idade mediana de ocorrência das fracturas atípicas (72 anos) foi, estatisticamente, menor do que nas fracturas típicas. Apesar de não se poder fazer uma comparação directa, esta distribuição etária parece estar de acordo ao apresentado por Meier et al que obteve a mediana das fracturas atípicas no grupo entre os 70-79 anos e das fracturas típicas no grupo entre os 80-89 anos. ${ }^{5}$ Ainda de acordo com este autor, o sexo feminino representa a maioria dos doentes em ambos os casos, com maior expressão nas fracturas atípicas (92\% vs $72 \%$, atípicas e típicas, respectivamente) ${ }^{5}$ Também esta distribuição é semelhante ao encontrado no nosso estudo com $100 \%$ de doentes do sexo feminino nas fracturas atípicas e $84 \%$ nas fracturas típicas.

O uso de bifosfonatos foi registado em seis (60\%) dos 10 doentes com fractura atípica. A percentagem foi sobreponível ao calculado por Park-Wyllie et $\mathrm{al}^{7}$ e inferior ao obtido por Thompson et al ${ }^{14}$ e Meier et al, ${ }^{5}$ respectivamente $64 \%, 81 \%$ e $92 \%$.

De acordo com o valor de odds ratio calculado no nosso trabalho de 101,1 (resultado estatisticamente significativo) num grupo com uma mediana de duração de tratamento de cinco anos, parece haver associação entre a ocorrência das fracturas atípicas e a toma de bifosfonatos. Nesta vertente, os nossos resultados são apoiados pela literatura com resultados de odds ratio de 9,46 em doentes com tratamento superior a três anos (Erviti et al) ${ }^{15}$; 66,9 em doentes com uma mediana de duração de tratamento no grupo entre os 5-9 anos (Meier et al) ${ }^{5}$; e 1000 em doentes com tratamento médio de 7,1 anos (Isaacs et al), ${ }^{6}$ resultados estes também estatisticamente significativos e com intervalos de confiança de $95 \%$.

Relativamente à presença de sintomas prodrómicos, Isaacs et al detectaram $71 \%$ dos doentes com dor prodrómica com uma duração entre três semanas e seis meses antes da fractura (tratamento médio de 7,1 anos). ${ }^{6} \mathrm{O}$ mesmo autor defende, se não a suspensão dos bifosfonatos ao fim de cinco anos, pelo menos a pesquisa activa de sintomas prodrómicos e monitorização radiográfica. No caso da nossa população em estudo estavam apenas descritos sintomas prodrómicos com uma duração de seis meses no caso da doente com fractura atípica bilateral simultânea.

Os pontos fortes do nosso estudo são a separação dos dois grupos (casos - atípicas vs controlos - típicas) com ocultação da informação do historial clínico e medicamentoso de cada doente; e o facto de termos utilizado os dados de todos os doentes elegíveis com os diagnósticos de fractura subtrocantérica e diafisária do fémur.

As principais limitações deste estudo foram o facto de 
se tratar de um estudo retrospectivo; o facto de ser apoiado por registos clínicos que podem não estar completos; a ausência de conhecimento de factores que podem alterar a massa óssea como o índice de massa corporal, história ginecológica ou medicação não activamente pesquisados; e o número pequeno de casos, mas que é inerente à raridade da situação clínica.

Este estudo deixa questões em aberto sendo a mais pertinente a razão pela qual ocorrem fracturas atípicas em doentes sem toma prévia de bifosfonatos. No nosso trabaIho detectámos quatro dos 10 doentes (40\%) com este tipo de fracturas sem antecedentes de toma de bifosfonatos. Destes, apenas dois tinham artrite reumatóide sob terapêutica com corticóides o que poderia contribuir para a fragilidade óssea, contudo ficam por explicar as restantes duas fracturas. São necessários mais estudos para compreender esta situação clínica na sua totalidade.

\section{CONCLUSÃO}

Apesar do pequeno número de casos foi possível demonstrar a relação, estatisticamente significativa, entre as fracturas femorais atípicas e a toma de bifosfonatos. De notar que estas fracturas atípicas ocorreram em doentes, significativamente, mais jovens do que os doentes com fracturas típicas.

Os resultados deste estudo advogam o uso criterioso dos bifosfonatos, sobretudo acima dos cinco anos.

\section{AGRADECIMENTOS}

Os autores gostariam de agradecer ao colega Joaquim G. Santos, especialista em Medicina Nuclear, pelo apoio prestado na análise estatística.

\section{CONFLITO DE INTERESSES}

Os autores declaram não haver nenhum conflito de interesses relativamente ao presente artigo.

\section{FONTES DE FINANCIAMENTO}

Os autores declaram não haver nenhuma fonte externa de financiamento para a realização deste artigo.

\section{REFERÊNCIAS}

1. Wells GA, Cranney A, Peterson J, Boucher M, Shea B, Welch V, et al. Alendronate for the primary and secondary prevention of osteoporotic fractures in postmenopausal women. Cochrane Database Syst Rev. 2008;CD001155.

2. Wells GA, Cranney A, Peterson J, Boucher M, Shea B, Welch V, et al. Risedronate for the primary and secondary prevention of osteoporotic fractures in postmenopausal women. Cochrane Database Syst Rev. 2008;CD004523.

3. Wells GA, Cranney A, Peterson J, Boucher M, Shea B, Welch V, et al. Etidronate for the primary and secondary prevention of osteoporotic fractures in postmenopausal women. Cochrane Database Syst Rev. 2008;CD003376.

4. Rizzoli R, Åkesson K, Bouxsein M, Kanis JA, Napoli N, Papapoulos $S$, et al. Subtrochanteric fractures after long-term treatment with bisphosphonates: a European Society on Clinical and Economic Aspects of Osteoporosis and Osteoarthritis, and International Osteoporosis Foundation Working Group Report. Osteoporos Int. 2011;22:373-90.

5. Meier RP, Perneger TV, Stern R, Rizzoli R, Peter RE. Increasing occurrence of atypical femoral fractures associated with bisphosphonate use. Arch Intern Med. 2012;172:930-6.

6. Isaacs JD, Shidiak L, Harris A, Szomor ZL. Femoral insufficiency fractures associated with prolonged bisphosphonate therapy. Clin Orthop Relat Res. 2010;468:3384-92.

7. Park-Wyllie LY, Mamdani MM, Juurlink DN, Hawker GA, Gunraj N, Austin PC, et al. Bisphosphonate use and the risk of subtrochanteric or femoral shaft fractures in older women. JAMA. 2011;305:783-9.
8. Shane E, Burr D, Abrahamsen B, Adler RA, Brown TD, Cheung AM, et al. Atypical subtrochanteric and diaphyseal femoral fractures: Second Report of a Task Force of the American Society for Bone and Mineral Research. J Bone Miner Res, 2014;29:1-24.

9. Odvina CV, Zerwekh JE, Sudhaker Rao D, Maalouf N, Gottschalk FA, Pak CYC. Severely suppressed bone turnover: a potential complication of alendronate therapy. J Clin Endocrinol Metab. 2005;90:1294-301.

10. Rosenberg ZS, Vieira RL, Chan SS, Babb J, Akyol Y, Rybak LD. Bisphosphonate-related complete atypical subtrochanteric femoral fractures: diagnostic utility of radiography. AJR. 2011;197:954-60.

11. BlacK DM, Kelly MP, Genant HK, Palermo L, Eastell R, Bucci-Rechtweg $\mathrm{C}$, et al. Bisphosphonates and fractures of the subtrochanteric or diaphyseal femur. N Engl J Med. 2010;362:1761-71.

12. Daniel WW. Biostatistics: a foundation for analysis in the health sciences. $7^{\text {th }}$ ed. John Wiley \& Sons; 1999

13. $\mathrm{R}$ Core Team. R: A language and environment for statistical, computing. $\mathrm{R}$ Foundation for Statistical Computing. Vienna, Austria; 2013; [consultado em 2013 Out 11], Disponível em http://www.R-project.org/.

14. Thompson RN, Phillips JR, McCauley SH, Elliott JR, Moran CG. Atypical femoral fractures and bisphosphonate treatment: experience in two large United Kingdom teaching hospitals. J Bone Joint Surg Br. 2012;94B:385-90.

15. Erviti, J, Alonso A, Oliva B, Gorricho J, López A, Timoner J, et al. Oral bisphosphonates are associated with increased risk of subtrochanteric and diaphyseal fractures in elderly women: a nested case-control study. BMJ Open. 2013;3:e002091. 


\section{Fracturas Femorais Atípicas e o Tratamento com Bifosfonatos: É um Factor de Risco?}

Acta Med Port 2014:27:704-709

Publicado pela Acta Médica Portuguesa, a Revista Científica da Ordem dos Médicos

Av. Almirante Gago Coutinho, 151

1749-084 Lisboa, Portugal.

Tel: +351218428215

E-mail: submissao@actamedicaportuguesa.com

www.actamedicaportuguesa.com

ISSN:0870-399X | e-ISSN: 1646-0758

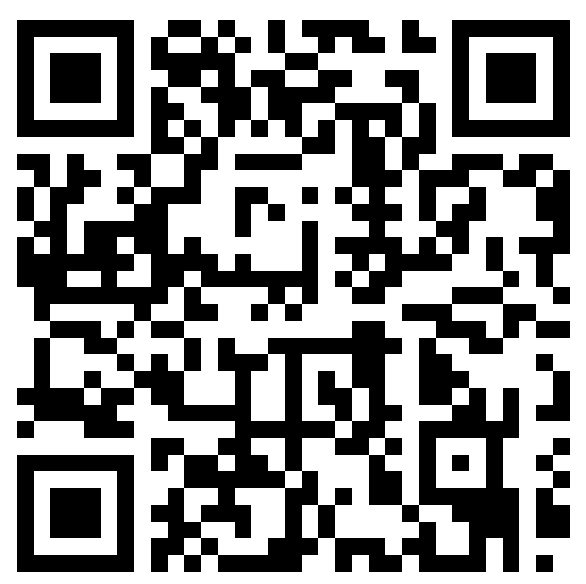

